\title{
La hiponatremia al momento de ingreso como factor de riesgo de mortalidad hospitalaria
}

\author{
JORGE VEGA ${ }^{1,2,4}$, FRANCISCO MANRÍQUEZ ${ }^{1,2}$, EVA MADRID ${ }^{3}$, \\ HELMUTH GOECKE ${ }^{1,2}$, ALEJANDRA CARRASCO ${ }^{1}$, \\ GONZALO MARTÍNEZ ${ }^{1,2}$, ALEJANDRO JOYAS ${ }^{5}$, FERNANDO ROJAS ${ }^{5}$, \\ JULIO SALINAS ${ }^{5}$, HERNÁN BORJA ${ }^{4}$
}

\section{Hyponatremia on admission to the emergency room as a risk factor for hospital mortality}

Background: Patients who develop hyponatremia during their hospitalization have higher hospital mortality. Aim: To determine if the presence of hyponatremia on admission to the emergency room is a risk factor for hospital mortality. Patients and Methods: Two hundred forty five patients consecutively admitted to the emergency room and then transferred to the Medicine Department, where they finally died, were matched for age and gender with 245 control subjects admitted to the emergency room and hospitalized in the Medicine Department at the same time, but survived. The dependent variable was death, and the exposure variable was hyponatremia. Admission diagnosis and Charlson comorbidity index was considered as confounding variables. Results: Hyponatremia at admission occurred in 30 and $17 \%$ of patients who died and survived, respectively, rendering an adjusted odds ratio for death of 2.13 (95\% confidence intervals $=1.27-3.57$ ). Charlson Comorbidity Index according to age score was higher in subjects with hyponatremia compared to those without hyponatremia $(8.1 \pm 3.1$ and $7.2 \pm 2.8 ; p=0.01)$. Multivariate analysis showed that age, gender, length of stay, cause of hospitalization and chronic diseases did not significantly modify the effect of hyponatremia on hospital mortality. Conclusions: Hyponatremia on admission at emergency room had a significant association with hospital mortality. Subjects with hyponatremia had a higher Charlson Comorbidity Index score.

(Rev Med Chile 2011; 139: 985-991).

Key words: Emergency Service, hospital; Hospital mortality; Hyponatremia.

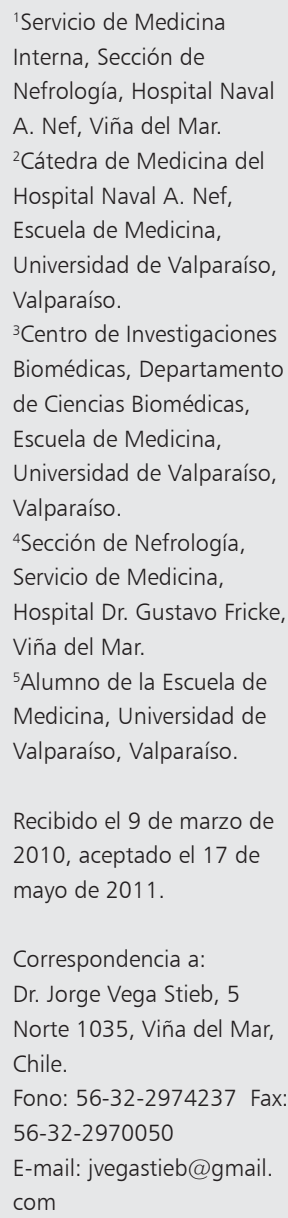

L a hiponatremia (HNA) es el trastorno hidroelectrolítico más frecuentemente observado comunicada de este trastorno es variable y depende de varios factores como: edad de la población estudiada, lugar de la atención médica, frecuencia con que son medidos los electrolitos, enfermedad que motivó la admisión al hospital, drogas en uso, puntos de corte en la definición de HNA, momento en el cual es determinada la natremia durante la hospitalización, etc ${ }^{1-17}$. La incidencia diaria de HNA en pacientes hospitalizados varía entre $0,97 \%$ y $1,5 \%$ y su prevalencia entre $2,5 \%$ y $2,6 \%{ }^{1-18}$. De todos los casos de HNA detectados durante la hospitalización, la proporción detectada en la admisión (hiponatremia adquirida en la comunidad) varía entre $25 \%$ y $63,8 \% 0^{1,17-19}$. Un estudio comunicó que $16 \%$ de los pacientes hospitalizados desarrollaba HNA durante su estadía y que en $46 \%$ de los que presentaban HNA en su admisión, esta se agravaba durante la hospitalización ${ }^{20}$. En las unidades de cuidados intensivos la 
incidencia de HNA varía entre $13,7 \%$ y $29,6 \%$ en el momento de la admisión ${ }^{21,22}$. La información acerca del significado de la presencia de HNA en el momento de admisión a un servicio de emergencia (SE) y su relación con la mortalidad hospitalaria es escasa ${ }^{12-14}$.

El objetivo de este trabajo, fue determinar si la presencia de HNA en el momento de admisión al hospital en el SE, se asociaba a mayor mortalidad hospitalaria.

\section{Sujetos y Métodos}

\section{Diseño del estudio}

Estudio analítico tipo caso-control desarrollado a objeto de establecer un modelo de asociación para determinar el efecto de la hiponatremia sobre la mortalidad hospitalaria. Se consideró variable de resultado a la muerte, la variable de exposición a la hiponatremia a la admisión y las variables de control fueron el índice de comorbilidad de Charlson corregido por edad $>6$, y los diferentes diagnósticos de ingreso (patología pulmonar infecciosa, patología infecciosa no pulmonar, accidentes cerebrovasculares, cardiopatías, insuficiencia renal, enfermedades respiratorias crónicas descompensadas, enfermedades digestivas, trombosis venosas profundas y/o embolia pulmonar, cáncer, enfermedades hepáticas, descompensación diabética y misceláneas).

Casos: 245 pacientes consecutivos admitidos a un SE y transferidos a un servicio de medicina interna, donde fallecieron.

Criterios de inclusión: Pacientes mayores de 15 años, de ambos géneros, portadores de patologías médicas, admitidos al hospital entre septiembre de 2005 y febrero de 2007 a través del SE con traslado posterior al servicio de medicina interna.

Criterios de exclusión: Pacientes trasladados desde el SE a las unidades de cuidados intensivos o servicios quirúrgicos, previo a su traslado al servicio de medicina. Sujetos que no contaron a su ingreso con determinaciones de natremia.

Grupo control: Cada caso fue pareado por género y edad con un paciente simultáneamente hospitalizado en el servicio de medicina (también admitido a través del SE), pero que sobrevivió la hospitalización.

Diecinueve $(7,7 \%)$ de los 245 pacientes fallecidos fueron excluidos por no contar con una determinación de natremia al ingreso, por lo que se excluyeron sus controles, dejando un número final de 452 pacientes.

Colección de datos: La siguiente información fue obtenida en los 452 pacientes incluidos en el estudio de sus historias clínicas y archivos computacionales: datos demográficos, duración de la hospitalización, mediciones séricas de sodio $\left(\mathrm{SNa}^{+}\right)$y glicemia en muestras obtenidas durante la admisión al SE y la condición vital al egresar del hospital. Se registró también la causa principal que motivó el ingreso al hospital y las patologías crónicas existentes. Con estas últimas se determinó el índice de comorbilidad de Charlson, el cual se ajustó por edad ${ }^{23}$.

Definiciones: Hiponatremia fue definida como $\mathrm{SNa}^{+} \leq 134 \mathrm{mEq} / \mathrm{L}$. La concentración de $\mathrm{SNa}^{+}$ fue corregida de acuerdo a la concentración de la glucosa al ingreso ${ }^{15}$.

\section{Análisis estadístico}

Se usó estadística paramétrica para evaluar asociaciones dada la gran cantidad de observaciones, basado en el teorema central del límite. El Odds Ratio fue la medida de riesgo con un intervalo de confianza de $95 \%$. Los datos fueron comparados con el uso del $\chi^{2}$, test exacto de Fischer o test $t$ de Student, según fuera apropiado. Los valores $<0,05$ fueron considerados como significativos para todos los análisis. Para el análisis multivariado se realizó el análisis de asociación cruda y la matriz de correlación para probar colinealidades. Luego se efectuó un análisis estratificado para cada una de las variables de control para buscar interacciones y se evaluó el sesgo de confusión. Finalmente, se generó el modelo de regresión logística multivariado y se probó la bondad de ajuste con prueba de $\chi^{2}$.

\section{Resultados}

No hubo diferencias significativas en el promedio de las natremias de ingreso, glicemias ni en la edad entre hombres y mujeres. Sus edades fueron 78,5 \pm 9,8 y 80,3 $\pm 11,4$ años; las natremias 
Hiponatremia como factor de riesgo de mortalidad - J. Vega et al

Tabla 1. Demografía y valores de natremia en la admisión al hospital

\begin{tabular}{|lcccc|}
\hline Pacientes & $\begin{array}{c}\text { Fallecidos } \\
\mathbf{n = 2 2 6}\end{array}$ & $\begin{array}{c}\text { Vivos } \\
\mathbf{n = 2 2 6}\end{array}$ & $\begin{array}{c}\text { Odds Ratio (IC 95\%) } \\
\text { No ajustados }\end{array}$ & p \\
\hline Edad: promedio (SD), años & $79,7(10.9)$ & $79,2(10,5)$ & & NS \\
\hline $\mathrm{Na}{ }^{+} \mathrm{mEq} / \mathrm{L}$, promedio (SD) & $138,4(9.3)$ & $138,7(6,3)$ & & $\mathrm{NS}$ \\
$\mathrm{Na}^{+} \leq 134 \mathrm{mEq} / \mathrm{L}, \%$ & 30,1 & 16,8 & $2,13(1,29-3,60)$ & 0,001 \\
$\mathrm{Na}^{+}>134-148 \mathrm{mEq} / \mathrm{L}, \%$ & 61,1 & 78,9 & $0,42(0,26-0,66)$ & 0,0001 \\
$\mathrm{Na}^{+} \geq 148 \mathrm{mEq} / \mathrm{L},(\%$ & 8,8 & 4,3 & $2,14(0,88-5,77)$ & $\mathrm{NS}$ \\
\hline Hospitalización, mediana (RIC), días & $8(5,18)$ & $14(9,24)$ & & 0,0001 \\
\hline Pacientes hiponatrémicos & $\mathbf{n}=\mathbf{6 8}$ & $\mathbf{n}=\mathbf{3 1}$ & & $\mathrm{NS}$ \\
\hline Edad: promedio (SD), años & $77,5(9.4)$ & $80,6(10.0)$ & & $\mathrm{NS}$ \\
\hline Hombres, \% & 51,5 & 41,9 & $1,47(0,57-3,80)$ & 0,002 \\
\hline Hospitalización, mediana (RIC), días & $9(5,23)$ & $15(11,25)$ & & $\mathrm{NS}$ \\
\hline Na ${ }^{+}<125 \mathrm{mEq} / \mathrm{L}, \%$ & 11,8 & 12,1 & $1,66(0,44-7,65)$ & \\
\hline
\end{tabular}

RIC: rango intercuartil.

de ingreso $137,9 \pm 7,5$ y $139,1 \pm 8,6 \mathrm{mEq} / \mathrm{L}$; y sus glicemias en la admisión $151,3 \pm 88,8$ y $162,6 \pm$ $107 \mathrm{mg} / \mathrm{dl}$, respectivamente.

La HNA fue más común en los pacientes que fallecieron durante la hospitalización, $30,1 \%$ vs $16,8 \%$. El análisis univariado mostró que la HNA al ingreso tuvo una asociación significativa con el riesgo de mortalidad hospitalaria, Odds Ratio (OR), 2,13 (Intervalo de confianza 95\%, 1,29-3,60) (Tabla 1). En los pacientes con HNA al ingreso no se encontraron diferencias significativas de edad y género entre sobrevivientes y fallecidos. Concentraciones de sodio $<125 \mathrm{mEq} / \mathrm{L}$ se observaron con una frecuencia similar en ambos grupos (Tabla 1).

Los sujetos con HNA que fallecieron, tuvieron estadías hospitalarias más breves que los que sobrevivieron (Tabla 1 ).

En los sujetos que fallecieron durante la hospitalización las neumonías como motivo de admisión y la presencia de comorbilidades neurológicas (Ej: demencia, enfermedad de Parkinson) o cáncer fueron más frecuentes que en los pacientes que sobrevivieron. El score en el índice de comorbilidad de Charlson fue significativamente superior en los fallecidos (Tabla 2).

En los pacientes con hiponatremia al ingreso fue más frecuente la presencia de hepatopatías crónicas y la descompensación de este trastorno como causal de admisión al hospital. También en ellos el score de comorbilidad de Charlson fue significativamente más elevado (Tabla 3).

Se hizo análisis estratificado de MantelHaenzel para las variables, índice de Charlson ajustado por edad de $>6$ puntos, las enfermedades gastrointestinales y las neumonías como causas de admisión. Una vez controlado el modelo, el Odds Ratio ajustado de la hiponatremia en la mortalidad fue 2,13 (IC 95\%, 1,27-3,57; $\mathrm{p}=0,004$ ).

\section{Discusión}

En nuestra serie 21,9\% de los pacientes fueron admitidos a la unidad de emergencia con HNA. Esta proporción de sujetos con HNA es superior a la comunicada previamente (12-14), lo que puede deberse al nivel de corte $(\leq 134 \mathrm{mEq} / \mathrm{L})$ escogido para definir HNA, el cual es superior al comúnmente utilizado $(<130 \mathrm{mEq} / \mathrm{L})$. Si nosotros hubiéramos utilizado este último nivel de corte, la incidencia de HNA hubiera sido 8,9\%. Una explicación adicional para esta disparidad, es que los pacientes que fueron transferidos directamente a las unidades de cuidados intensivos no fueron incluidos en esta serie. Un ejemplo de ellos fueron los pacientes ingresados por un infarto agudo al miocardio, los cuales por el escaso intervalo entre el inicio de los síntomas y su admisión, es muy probable que no hayan tenido el tiempo suficiente para desarrollar HNA. Si ellos hubieran sido 
Tabla 2. Frecuencia de enfermedades crónicas y causas de ingreso al hospital en sobrevivientes y fallecidos

\begin{tabular}{|c|c|c|c|c|}
\hline Enfermedades crónicas & $\begin{array}{l}\text { Fallecidos } \\
(n=226)\end{array}$ & $\begin{array}{c}\text { Vivos } \\
(n=226)\end{array}$ & Odds Ratio (IC 95\%) & $\mathbf{p}$ \\
\hline E. Cardíacas (\%) & 36,0 & 35,9 & $1,00(0,66-1,53)$ & 0,98 \\
\hline E. Cerebrovasculares (\%) & 21,3 & 18,0 & $1,24(0,742,07)$ & 0,39 \\
\hline E. Respiratorias (\%) & 14,2 & 20,4 & $0,65(0,37-1,12)$ & $0,01 *$ \\
\hline E. Neurológicas (\%) & 56,9 & 37,9 & $2,16(1,43-3,26)$ & 0,0001 * \\
\hline Diabetes Mellitus (\%) & 26,1 & 31,1 & $0,78(0,50-1,22)$ & 0,26 \\
\hline E. Renales (\%) & 15,6 & 18,9 & $0,79(0,46-1,36)$ & 0,37 \\
\hline E. Hepáticas (\%) & 10,9 & 6,3 & $1,81(0,85-4,02)$ & 0,95 \\
\hline E. Gastrointestinales (\%) & 3,8 & 3,9 & $0,97(0,31-3,04)$ & 0,96 \\
\hline Cáncer (\%) & 39,8 & 28,6 & $1,65(1,07-2,53)$ & $0,01^{*}$ \\
\hline E. Colágeno-vasculares (\%) & 1,4 & 3,9 & $0,36(0,06-1,52)$ & 0,11 \\
\hline Hipotiroidismo (\%) & 9,9 & 12,1 & $0,80(0,41-1,55)$ & 0,48 \\
\hline I.C.Charlson, media (DS) & $4,25(2,65)$ & $3,51(2,67)$ & & $0,004^{*}$ \\
\hline Charlson Aj. media (DS) & $7,75(2,74)$ & $6,92(2,99)$ & & $0,003^{*}$ \\
\hline \multicolumn{5}{|l|}{ Causas de ingreso } \\
\hline Neumonías (\%) & 27,7 & 15,5 & $2,09(1,26-3,50)$ & $0,002^{*}$ \\
\hline Otras infecciones (\%) & 13,1 & 14,0 & $0,92(0,51-1,68)$ & 0,80 \\
\hline Acc. cerebrovasculares (\%) & 8,4 & 7,2 & $1,18(0,54-2,59)$ & 0,65 \\
\hline Cardiopatías (\%) & 8,4 & 10,6 & $0,78(0,38-1,57)$ & 0,45 \\
\hline Insuficiencia renal (\%) & 3,3 & 3,9 & $0,84(0,25-2,72)$ & 0,75 \\
\hline Enf. respiratorias (\%) & 3,8 & 8,7 & $0,41(0,15-1,02)$ & $0,035^{*}$ \\
\hline Enf. digestivas (\%) & 4,2 & 14,0 & $0,27(0,11-0,61)$ & $0,0005^{*}$ \\
\hline TVP-ETE (\%) & 1,4 & 1,0 & $1,46(0,16-17)$ & 0,68 \\
\hline Cáncer (\%) & 17,4 & 11,6 & $1,60(0,89-2,92)$ & 0,093 \\
\hline E. Hepáticas (\%) & 5,2 & 2,4 & $2,20(0,69-8,21)$ & 0,14 \\
\hline Descompensación DM (\%) & 3,4 & 5,3 & $0,69(0,23-1,94)$ & 0,44 \\
\hline Misceláneas (\%) & 3,3 & 5,8 & $0,55(0,18-1,56)$ & 0,22 \\
\hline
\end{tabular}

TVP-ETE: trombosis venosa profunda/enfermedad tromboembólica pulmonar. Acc: accidentes. DM: diabetes mellitus. I.C. Charlson: score del Índice de Comorbilidad de Charlson. Aj: ajustado por edad.

incluidos, la proporción de enfermos con HNA al ingreso hubiera sido menor.

La información disponible respecto a la incidencia de HNA en los pacientes internados en una unidad de emergencia es escasa. Algunos autores han comunicado una incidencia entre 3,8\% y $4,0 \%{ }^{12-14}$.

Existe más información respecto a su asociación a patologías específicas como insuficiencia cardiaca, cirrosis hepática, neumonía adquirida en la comunidad e infección por VIH. La HNA en pacientes admitidos con insuficiencia cardiaca es común y varía entre $19,7 \%$ y $27,0 \% \%^{2,3,24,25}$. En pacientes con otras patologías como cirrosis hepática, este trastorno está presente entre $29,8 \%$ y $34,8 \% 5,6$, en la neumonía adquirida en la comunidad $23,0 \%{ }^{26}$ y en los pacientes infectados con VIH $21,6 \%{ }^{7}$. En nuestra serie la hiponatremia fue 
Tabla 3. Frecuencia de enfermedades crónicas y causas de ingreso al hospital en pacientes hiponatrémicos y no-hiponatrémicos

\begin{tabular}{|c|c|c|c|c|}
\hline Enfermedades crónicas & $\begin{array}{l}\text { Hiponatrémicos } \\
(\mathbf{n}=99)\end{array}$ & $\begin{array}{l}\text { No hiponatrémicos } \\
(\mathrm{n}=353)\end{array}$ & $\begin{array}{l}\text { Odds Ratio } \\
\text { (IC 95\%) }\end{array}$ & $\mathbf{p}$ \\
\hline E. Cardíacas (\%) & 37,6 & 37,7 & $0,99(0,59-1,66)$ & 0,99 \\
\hline E. Cerebrovasculares (\%) & 22,6 & 20,4 & $1,14(0,61-2,05)$ & 0,66 \\
\hline E. Respiratorias (\%) & 12,9 & 19,7 & $0,60(0,28-1,21)$ & 0,14 \\
\hline E. Neurológicas (\%) & 43,0 & 52,8 & $0,67(0,40-1,10)$ & 0,10 \\
\hline Diabetes Mellitus (\%) & 25,8 & 29,6 & $0,82(0,46-1,44)$ & 0,49 \\
\hline E. Renales (\%) & 20,4 & 15,5 & $1,40(0,72-2,62)$ & 0,27 \\
\hline E. Hepáticas (\%) & 17,2 & 5,6 & $3,48(1,54-7,78)$ & $0,0005^{*}$ \\
\hline E. Gastrointestinales (\%) & 1,1 & 4,6 & $0,22(005-1,55)$ & 0,12 \\
\hline Cáncer (\%) & 39,8 & 32,0 & $1,40(0,83-2,33)$ & 0,17 \\
\hline E. Colágeno-vasculares (\%) & 1,0 & 2,8 & $0,37(0,008-2,86)$ & 0,34 \\
\hline Hipotiroidismo (\%) & 10,7 & 11,6 & $0,92(0,38-2,00)$ & 0,82 \\
\hline I.C.Charlson, media (DS) & $4,73(2,95)$ & $3,71(2,55)$ & & $0,001^{*}$ \\
\hline Charlson Aj. media (DS) & $8,10(3,10)$ & $7,22(2,84)$ & & 0,01 * \\
\hline \multicolumn{5}{|l|}{ Causas de ingreso } \\
\hline Neumonías (\%) & 21,3 & 22,7 & $0,92(0,49-1,66)$ & 0,77 \\
\hline Otras infecciones (\%) & 18,1 & 12,2 & $1,58(0,78-3,08)$ & 0,15 \\
\hline Acc. cerebrovasculares (\%) & 6,4 & 9,1 & $0,68(0,22-1,76)$ & 0,41 \\
\hline Cardiopatías (\%) & 7,4 & 11,5 & $0,62(0,22-1,48)$ & 0,26 \\
\hline Insuficiencia renal (\%) & 3,2 & 3,2 & $1,01(0,17-4,18)$ & 0,98 \\
\hline Enf. respiratorias (\%) & 2,1 & 7,7 & $0,26(0,03-1,09)$ & 0,054 \\
\hline Enf. digestivas (\%) & 5,3 & 8,7 & $0,59(0,17-1,62)$ & 0,29 \\
\hline TVP-ETE (\%) & 2,1 & 0,7 & $3,08(0,22-42)$ & 0,24 \\
\hline Cáncer (\%) & 18,9 & 13,3 & $1,44(0,72-2,78)$ & 0,25 \\
\hline E. Hepáticas (\%) & 10,6 & 1,8 & $6,69(2,0-25,5)$ & 0,0001 * \\
\hline Descompensación DM (\%) & 4,3 & 4,9 & $0,86(0,20-2,84)$ & 0,80 \\
\hline Misceláneas (\%) & 1,0 & 4,2 & $0,24(0,005-1,70)$ & 0,15 \\
\hline
\end{tabular}

TVP-ETE: trombosis venosa profunda/ enfermedad tromboembólica pulmonar. Acc: accidentes. DM: diabetes mellitus. I.C. Charlson: score del Índice de Comorbilidad de Charlson. Aj: ajustado por edad.

significativamente más frecuente en los sujetos aquejados de un daño hepático crónico y en los admitidos al hospital por una descompensación de esta patología.

La edad de los pacientes con HNA no fue diferente a la de los admitidos con una natremia normal (78,5 vs 80 años). Algunos autores han comunicado que la frecuencia de HNA aumenta a medida que la edad de los pacientes es mayor. Un estudio, usando como referencia a mujeres $<$ de 30 años de edad, encontró que el Odds Ratio para sujetos mayores de 80 años fue 1,89 y 8,70 , para natremias $<136 \mathrm{mEq} / \mathrm{L} \mathrm{y}<116 \mathrm{mEq} / \mathrm{L}$, respectivamente $^{19}$. Un estudio prospectivo, efectuado en pacientes hospitalizados, encontró que los pacientes con HNA fueron 8 años mayores que los con natremia normal, no encontrando diferencias en su género ${ }^{14}$. En otra comunicación ${ }^{19}$ el género no 
fue un factor de riesgo de HNA, tampoco lo fue en la nuestra.

En esta serie, la presencia de HNA en el momento de la admisión (hiponatremia adquirida en la comunidad) fue más frecuente en los sujetos que fallecieron que en los que sobrevivieron $(30,1 \%$ vs $16,8 \%)$. En otro estudio, diseñado para evaluar el riesgo de muerte de pacientes con HNA a la admisión, los autores encontraron que el riesgo era 7 veces mayor que los sujetos con natremia normal, pareados por sexo, edad y fecha de admisión. Ellos concluyeron que la HNA en la admisión era un factor de riesgo de muerte intrahospitalaria, independientemente de las enfermedades a las que estaba asociada ${ }^{13}$. Este mismo hallazgo fue encontrado por nosotros en este grupo de pacientes. Una publicación reciente de una investigación efectuada en 53.236 hospitalizaciones, comunicó una incidencia de hiponatremia en la admisión de $37,9 \%$. Ella se acompañó de una mortalidad superior a la de los sujetos que ingresaron sin el trastorno (3,4\% vs 2,0\%; Odds Ratio ajustado: 1,66 $\left(95 \%\right.$ CI, 1,39-1,98) ${ }^{27}$. En otras comunicaciones acerca de la mortalidad hospitalaria de los pacientes con patologías médicas e HNA, admitidos a un servicio de urgencia, la mortalidad ha variado entre $17,9 \%$ y $27 \%{ }^{12,14}$.

Se ha comunicado que la severidad de la HNA se asocia a una mayor mortalidad hospitalaria $^{12,18,27}$. En nuestra serie, aunque los niveles de natremia $<125 \mathrm{mEq} / \mathrm{L}$ no fueron más frecuentes en los fallecidos que en los sobrevivientes, $66,7 \%$ de los que tuvieron natremias $<125 \mathrm{mEq} / \mathrm{L}$ fallecieron. La mortalidad de los pacientes con HNA internados en un servicio de medicina es 3 veces más alta que la de los pacientes sin el trastorno. La tasa de mortalidad puede alcanzar hasta $62 \%$ cuando la natremia es $<115 \mathrm{mEq} / \mathrm{L}^{9-11}$.

En esta serie, si bien los pacientes con hiponatremia al ingreso no difirieron respecto a los sin hiponatremia en la mayoría de los motivos de admisión al hospital o en la prevalencia de las enfermedades crónicas más frecuentes, salvo las hepatopatías crónicas, el número de patologías asociadas en ellos fue superior (utilizando el score de comorbilidad de Charlson).

Una de las limitaciones de este estudio fue el haber tenido que eliminar a algunos pacientes por no haber contado con una determinación de natremia al ingreso. Sin embargo, el número de sujetos excluidos fue pequeño ( 38 de 490 ) por lo que es improbable que ello haya influido en los resultados.

En suma, en esta serie de pacientes con patologías médicas, la existencia de HNA al ingreso al hospital a través del servicio de emergencia fue más común en los sujetos que no sobrevivieron la hospitalización. Por ello, la HNA (corregida según la glicemia) puede considerarse como un marcador de riesgo de muerte intrahospitalaria.

\section{Referencias}

1. Anderson R, Chung H, Kluge R, Schrier R. Hyponatremia: a prospective analysis of its epidemiology and the pathogenic role of vasopressin. Ann Intern Med 1985; 102: $164-8$.

2. Gheorghiade M, Abraham W, Albert N, Gattis-Stough W, Greenberg B, O'Coonor C, et al. Relationship between admission serum sodium concentration and clinical outcomes in patients hospitalized for heart failure: an analysis from OPTIMIZE-HF registry. Eur Heart J 2007; 28: 980-8.

3. Klein L, O'Connor C, Leimberger J, Gattis-Stough W, Piña I, Felker M, et al. Lower serum sodium is associated with increased short-term mortality in hospitalized patients with worsening heart failure. Circulation 2005; 111: 2454-60.

4. Lee D, Austin P, Rouleau J, Liu P, Naimark D, Tu J. Predicting mortality among patients hospitalized for heart failure. Derivation and validation of a clinical model. JAMA 2003; 290: 2581-7.

5. Borroni G, Maggi A, Sangiovanni A, Cazzaniga M, Salerno F. Clinical relevance of hyponatremia for the hospital outcome of cirrhotic patients. Dig Liver Dis 2000; 32: 605-10.

6. Porcel A, Díaz F, Rendón P, Macías M, Martín-Herrera L, Girón-González J. Dilutional hyponatremia in patients with cirrhosis and ascites. Arch Intern Med 2002; 162: 323-8.

7. Tang W, Kaptein E, Feinstein E, Massry S. Hyponatremia in hospitalized patients with the acquired immunodeficiency syndrome (AIDS) and the AIDS-related complex. Am J Med 1993; 94: 169-74.

8. Cusano A, Thies H, Siegal F, Dreisbach A, Maesaka J. Hyponatremia in patients with acquired immune deficiency syndrome. J Acquir Immune Defic Syndr 1990; 3: 949-53.

9. Clayton J, Le Jeune I, Hall I. Severe hyponatremia in medical in-patients: aetiology, assessment and outcome. QMJ 2006; 99: 505-11. 
10. Erasmus R, Matsha T. The frequency, aetiology and outcome of severe hyponatremia in adult hospitalized patients. Cent Afr J Med 1998; 44: 154-8.

11. Baran D, Hutchinson T. The outcome of hyponatremia in a general hospital population. Clin Nephrol 1984; 22: 72-6.

12. Lee $\mathrm{C}$, Guo H, Chen J. Hyponatremia in the emergency department. Am J Emerg Med 2000; 18: 264-8.

13. Tierney W, Martin D, Greenlee M, Zerbe R, McDonald C. The prognosis of hyponatremia at hospital admission. J Gen Intern Med 1986; 1: 380-5.

14. Gill G, Huda B, Boyd A, Skagen K, Wile D, Watson I, et al. Characteristics and mortality of severe hyponatremia-a hospital-based study. Clin Endocrinol (Oxf) 2006; 65: 246-9.

15. Hillier TA, Abbott RD, Barrett EJ. Hyponatremia: Evaluating the correction factor for hyperglicemia. Am J Med 1999; 106: 399-403.

16. Upadhyay A, Jaber B, Madias N. Incidence and prevalence of hyponatremia. Am J Med 2006; 119: S30-5.

17. Natkunam A, Shek C, Swaminathan R. Hyponatremia in a hospital population. J Med 1991; 22: 83-96.

18. Pizzotti N, Madi J, Iamanaca A, Seguro A, Rocha A. Hyponatremia: study of its epidemiology and mortality. Rev Hosp Clin Fac Med Sao Paulo 1989; 44: 307-11.

19. Hawkins R. Age and gender as risk factors for hyponatremia and hypernatremia. Clin Chim Acta 2003; 337: 169-72.

20. Hoorn E, Lindermans J, Zietse R. Hyponatremia in hospitalized patients: epidemiology, etiology and symptomatology (abstract). J Am Soc Nephrol 2004; 15: 561 A.

21. Bennani S, Abougal R, Zeggwagh A, Madani N, Abidi K, Zekraoul A, et al. Incidence, causes and prognostic factors of hyponatremia in intensive care. Rev Med Interne 2003; 24: 224-9.

22. DeVita M, Gardenswartz M, Konecky A, Zabetakis P. Incidence and etiology of hyponatremia in an intensive care unit. Clin Nephrol 1990; 34: 163-1.

23. Charlson ME, Pompei P, Ales KL, MacKenzie CR. A new method of classifying prognostic comorbidity in longitudinal studies: development and validation. J Chron Dis 1987; 40: 373-83.

24. Gheorghiade M, Gattis-Stough W, O'Connor C, Adams K, Elkayam U, Barbagelata A, et al. Effects of Tolvaptan, a vasopressin antagonist, in patients hospitalized with worsening heart failure. JAMA 2004; 291: 1963-71.

25. Gheorghiade M, Rossi J, Cotts W, Shin D, Hellkamp A, Piña I, et al. Characterization and prognostic value of persistent hyponatremia in patients with severe heart failure in the ESCAPE trial. Arch Intern Med 2007; 167: 1998-2005.

26. Torres J, Cardenas O, Vásquez A, Schlossberg D. Streptococcus pneumoniae bacteremia in a community hospital. Chest 1998; 113: 387-90.

27. Wald R, Jaber BL, Price LL, Upadhyay A, Madias NE. Impact of hospital-associated hyponatremia on selected outcomes. Arch Intern Med 2010; 170: 294-302. 